\title{
INTERFACE SEPARATION IN THE TRANSONIC RANGE CAUSED BY A PLANE STRESS PULSE
}

\author{
M. Comninou \\ Department of Applied Mechanics and Engineering Science, \\ University of Michigan, Ann Arbor, Michigan 48109, U.S.A. \\ AND

\section{J. Dundurs} \\ Department of Civil Engineering, \\ Northwestern University, Evanston, Illinois 60201, U.S.A.
}

(Received 6 May 1978)

\begin{abstract}
This is a continuation of the authors' investigation [1] of the localized separation between two contacting solids that is caused by an incident plane elastic wave of arbitrary form. The interface is taken as frictionless and incapable of transmitting tensile tractions. It is further assumed that the two solids have identical mechanical properties, and that the angle of incidence of the incoming wave is such that the disturbance propagates along the interface with a transonic speed. Moving dislocations are employed in the formulation and the problem is reduced to a singular integral equation with a Cauchy kernel. Specific results are worked out for an incident tensile stress pulse of a parabolic shape. An intriguing aspect of the results is that, in spite of the incident wave being continuous, the elastic fields are singular at the trailing edge of the separatior. zone.
\end{abstract}

\section{INTRODUCTION}

We continue in this article our study of the separation between two solids that is caused by an incident plane elastic wave of arbitrary form [1]. The interface is still taken as frictionless, but the angle of incidence of the incoming wave is allowed to be such that the disturbance propagates along the interface with a speed that is no longer supersonic, and the process involves total reflection. As with harmonic incident waves $[2,3]$, a large number of cases must be considered individually when the materials are different. Our present investigation is for this reason restricted to identical materials. Nonetheless, some salient features of the interaction between a unilateral interface and an incident wave will emerge.

Suppose that the phase velocity of the incident wave is $c_{0}$ and the angle of incidence $0_{0}$. The disturbance then propagates along the interface with the velocity

$$
v=c_{0} / \sin \theta_{0} \text {. }
$$

If this velocity is to fall in the transonic range $\left(c_{T}<v<c_{L}\right)$ for identical materials, the incident wave causing separation must be a vertically polarized shear (SV) wave. As in reference [1], the solution for the unilateral interface is constructed by cancelling the tensile tractions in the bilateral problem. This is done by relating a gap that propagates along the interface to a distribution of dislocations and taking advantage of some known results for moving dislocations. 


\section{FORMULATION}

Consider first the bilateral problem in which the frictionless contact interface is allowed to transmit tensile tractions, with the same notation and placement of co-ordinate axes as in reference [1]. If the trace or apparent phase velocity $v$ given by equation (1) is in the transonic range, the reflected and refracted $P$-waves are trapped at the interface and assume the form of surface waves. In addition, the reflected and refracted SV-waves become distorted $[4,5]$. However, the resultant normal tractions that are transmitted by the bilateral interface retain the original shape of the normal tractions imposed by the incident wave:

$$
S(\eta)=\mathscr{A}_{0} f(\eta), \quad \mathscr{A}_{0}=C_{0} k_{2} \mu \sin 2 \theta_{2},
$$

where $f(\eta)$ is related to the incident wave by equation (6) in reference [1]. This peculiarity is due to the fact that the amplitude $\mathscr{A}_{0}$ of the bilateral normal tractions remains real for identical materials although the reflection and transmission coefficients become complex.

In order to cancel the tensile tractions in the bilateral problem and allow for separation between the two solids, the separation zones may be viewed as arrays of edge dislocations that move by climb with a transonic speed $v$. As mentioned in reference [1], it is expedient to break the total dislocation distribution, $B(\eta)$, into four parts connected with the reflected and refracted waves in the two solids:

$$
B(\eta)=B_{L}(\eta)+B_{T}(\eta)+\bar{B}_{L}(\eta)+\bar{B}_{T}(\eta)
$$

The interface tractions induced by each of the four distributions moving in the transonic range $\left(c_{T}<v<c_{L}\right)$ can be extracted from the formulas given by Berg et al. [6]. Thus

$$
\begin{aligned}
& \tau_{x y}(\eta, 0)=\mu\left\{2 B_{L}(\eta)-\left(\zeta_{T}^{2}-1\right) B_{T}(\eta)\right\}, \\
& \tau_{y y}(\eta, 0)=\mu\left\{\frac{\zeta_{T}^{2}-1}{\pi \zeta_{L}^{*}} \int_{-\infty}^{\infty} \frac{B_{L}(\xi) \mathrm{d} \xi}{\xi-\eta}-2 \zeta_{T} B_{T}(\eta)\right\}, \\
& \bar{\tau}_{x y}(\eta, 0)=\mu\left\{-2 \bar{B}_{L}(\eta)+\left(\zeta_{T}^{2}-1\right) \bar{B}_{T}(\eta)\right\}, \\
& \bar{\tau}_{y y}(\eta, 0)=\mu\left\{\frac{\zeta_{T}^{2}-1}{\pi \zeta_{L}^{*}} \int_{-\infty}^{\infty} \frac{\bar{B}_{L}(\xi) \mathrm{d} \xi}{\xi-\eta}-2 \zeta_{T} \bar{B}_{T}(\eta)\right\},
\end{aligned}
$$

where

$$
\zeta_{L}^{*}=\left(1-v^{2} / c_{L}^{2}\right)^{1 / 2}, \quad \zeta_{T}=\left(v^{2} / c_{T}^{2}-1\right)^{1 / 2}
$$

As in reference [1], the four distributions now can be adjusted so that the normal tractions are continuous and the shearing tractions vanish at the interface. This yields

$$
\begin{aligned}
& B_{L}(\eta)=\bar{B}_{L}(\eta)=\left\{\left(\zeta_{T}^{2}-1\right) / 2\left(\zeta_{T}^{2}+1\right)\right\} B(\eta), \\
& B_{T}(\eta)=\bar{B}_{T}(\eta)=\left\{1 /\left(\zeta_{T}^{2}+1\right)\right\} B(\eta)
\end{aligned}
$$

and the normal tractions induced by the total dislocation distribution become

$$
\begin{aligned}
T(\eta) & =\tau_{y y}(\eta, 0)=\bar{\tau}_{y y}(\eta, 0) \\
& =-\frac{\mu}{2\left(\zeta_{T}^{2}+1\right)}\left\{4 \zeta_{T} B(\eta)-\frac{\left(\zeta_{T}^{2}-1\right)^{2}}{\pi \zeta_{L}^{*}} \int_{-\infty}^{\infty} \frac{B(\zeta) \mathrm{d} \xi}{\xi-\eta}\right\}, \quad-\infty<\eta<\infty .
\end{aligned}
$$

The condition which remains to be enforced is that the resultant normal tractions

$$
N(\eta)=S(\eta)-p^{\infty}+T(\eta)
$$


vanish in the separation zones. Since the normal tractions are no longer determined merely by the local value of the dislocation density $B(\eta)$, as they were in the supersonic case, but involve the whole array because of the integral in (11), the problem is considerably more complicated than before.

One can begin by considering an incident stress pulse that leads to a single separation zone $\alpha<\eta<\beta$ in the moving co-ordinate system. $B(\eta)$ is then zero outside the interval $\alpha<\eta<\beta$. The requirement that $N(\eta)=0$ in the separation zone leads to the integral equation

$$
B(\eta)+\frac{b}{\pi} \int_{\alpha}^{\beta} \frac{B(\xi) \mathrm{d} \xi}{\xi-\eta}=\frac{\zeta_{T}^{2}+1}{2 \mu \zeta_{T}}\left[\mathscr{A}_{0} f(\eta)-p^{\infty}\right], \quad \alpha<\eta<\beta,
$$

where

$$
b=-\left(\zeta_{T}^{2}-1\right)^{2} / 4 \zeta_{L}^{*} \zeta_{T}
$$

The general solution of equation (13) is given in reference [7] as

$$
B(\eta)=\frac{\mathscr{A}_{0}\left(1+\zeta_{T}^{2}\right)}{2 \mu \zeta_{T}\left(1+b^{2}\right)}\left\{f(\eta)-\frac{p^{\infty}}{\mathscr{A}_{0}}+C w(\eta)-\frac{b}{\pi} w(\eta) \int_{\alpha}^{\beta} \frac{f(\xi)-\left(p^{\infty} / \mathscr{A}_{0}\right)}{(\xi-\eta) w(\xi)} \mathrm{d} \xi\right\},
$$

where $C$ is a non-zero constant for solutions singular at both ends, $\eta=\alpha$ and $\eta=\beta$, of the separation zone, and $w(\eta)$ is the characteristic function of the singular integral equation. Its exact form depends on the behavior of $B(\eta)$ at the ends of the separation interval.

In order to avoid a global discontinuity in the displacements, the total dislocation must vanish, or

$$
\int_{\alpha}^{\beta} B(\xi) \mathrm{d} \xi=0
$$

The physical inequalities

$$
g(\eta) \geqslant 0
$$

in the separation zone and

$$
N(\eta) \leqslant 0
$$

in the contact zones must also be verified. In expression $(17), g(\eta)$ denotes the gap between the solids, which is related to the dislocation density by

$$
B(\eta)=-\mathrm{d} g(\eta) / \mathrm{d} \eta \text {. }
$$

One can now look first for a solution $B(\eta)$ that is bounded at both ends of the separation interval and satisfies condition (16). Then, from reference [7],

$$
C=0, \quad w(\eta)=(\beta-\eta)^{A}(\eta-\alpha)^{1-A},
$$

where

$$
A=(1 / \pi) \tan ^{-1}(-b), \quad 0<A<\frac{1}{2} .
$$

In addition, the following consistency condition must be satisfied:

$$
\int_{\alpha}^{\beta} \frac{f(\xi)-\left(p^{\infty} / \mathscr{A}_{0}\right)}{w(\xi)} \mathrm{d} \xi=0
$$

It may be noted that equations (16) and (23) provide two conditions for the determination of the unknown parameters $\alpha$ and $\beta$. Substituting $B(\eta)$ from equation (15) into equation 
(16) and using equations (20)-(23) leads to, after some integrations,

$$
\int_{\alpha}^{\beta} \frac{\xi\left[f(\xi)-\left(p^{\infty} / \mathscr{A}_{0}\right)\right]}{w(\xi)} \mathrm{d} \zeta=0 .
$$

It can be anticipated from the results for harmonic waves [3], however, that solutions singular at the trailing edge, $\eta=\alpha$, of the separation zone may have to be admitted. While expressions (20) and (22) remain valid for such solutions, the characteristic function then changes to

$$
w(\eta)=(\beta-\eta)^{A}(\eta-\alpha)^{-A}
$$

and the consistency condition (23) must be discarded. It follows from equations (22) and (25) that the singularity is weaker than the inverse square root. Equation (16) yields for this case

$$
\int_{\alpha}^{\beta} \frac{f(\xi)-\left(p^{\infty} / \mathscr{A}_{0}\right)}{w(\xi)} \mathrm{d} \xi=0 .
$$

Although condition (26) appears to be identical with condition (23), the two are not the same because the characteristic functions (21) and (25) which must be substituted into them are different.

At this stage, the shape of the incident stress pulse must be specified in order to proceed with the solution.

\section{PARABOLIC PULSE}

As an example, it is convenient to consider the parabolic tension pulse

$$
f(\eta)=\left\{\begin{array}{ll}
1-\eta^{2}, & |\eta| \leqslant 1 \\
0, & |\eta| \geqslant 1
\end{array}\right\},
$$

which has also been discussed for supersonic speeds of the separation zone [1]. In the course of the solution, one must distinguish between two cases: at relatively high levels of the applied pressure $p^{\infty}$ (Case I), the separation zone lies entirely within the interval $(-1,1)$; for low levels of $p^{\infty}$ (Case II), the separation zone may extend beyond $\eta=-1$. Case I can be solved in closed form, but numerical computations are required for Case II.

\subsection{CASE I}

For the bounded solution, instead of specifying $p^{\infty} / \mathscr{A}_{0}$ and then determining $\alpha$ and $\beta$, it is simpler to assign values to $\alpha$ and to solve for the other two parameters. Thus, from equations (23) and (27), after some elementary integrations,

$$
p^{\infty} / \alpha_{0}=1-\alpha^{2}-2 A \alpha(\beta-\alpha)-\frac{1}{2} A(1+A)(\beta-\alpha)^{2}
$$

and, from equations (24), (27) and (28),

$$
\beta=-\{(2-A) /(1+A)\} \alpha, \quad \alpha \leqslant 0 .
$$

With $B$ given by equation (29), equation (28) becomes

$$
p^{\infty} / \mathscr{A}_{0}=1-\{(2-A) / 2(1+A)\} \alpha^{2} .
$$


Finally, one obtains, from equation (15)

$$
B(\eta)=-\frac{\mathscr{A}_{0}\left(1+\zeta_{T}^{2}\right) \cos \pi A}{2 \mu \zeta_{T}} w(\eta)\left(\eta+\frac{1-2 A}{1+A} \alpha\right), \quad \alpha<\eta<\beta .
$$

It can easily be shown from equations (31) and (19) that the resulting gap is negative and, therefore, condition (17) is not satisfied.

A similar situation was encountered for incident harmonic waves and separation zones propagating in the transonic range [3]. The difficulty in reference [3] was resolved by admitting integrable singularities at the trailing edges of the separation zones. The same approach is adopted here.

Substituting equations (25) and (27) into equation (26) and performing the elementary integrations gives

$$
p^{\infty} / \mathscr{A}_{0}=1-\alpha^{2}-(1+A) \alpha(\beta-\alpha)-\frac{1}{6}(1+A)(2+A)(\beta-\alpha)^{2} .
$$

The dislocation distribution follows from equation (15) as

$$
\begin{aligned}
B(\eta)= & \left\{\mathscr{A}_{0}\left(1+\zeta_{T}^{2}\right) \cos (\pi A) / 2 \mu \zeta_{T}\right\} w(\eta) \\
& \times\left\{\alpha \beta+\frac{1}{3}\left(1-A^{2}\right)(\beta-\alpha)^{2}-A(\beta-\alpha) \eta-\eta^{2}\right\}, \quad \alpha<\eta<\beta .
\end{aligned}
$$

It can casily be shown from equation (33) that condition (17) is satisfied if

$$
\beta \leqslant-\{(1-A) /(2+A)\} \alpha .
$$

Next, with $B(\eta)$ given by equation (33), the normal tractions in the contact zones are, from equations (12) and (11),

$$
\begin{gathered}
N(\eta)=-\mathscr{A}_{0} \hat{f}(\eta)-\mathscr{A}_{0}|(\eta-\beta) /(\eta-\alpha)|^{A}\left\{\eta^{2}+A(\beta-\alpha) \eta-\alpha \beta-\frac{1}{3}\left(1-A^{2}\right)(\beta-\alpha)^{2}\right\} \\
-\infty<\eta<\alpha \text { and } \beta<\eta<\infty
\end{gathered}
$$

where

$$
\hat{f}(\eta)=\left\{\begin{array}{ll}
0, & |\eta| \leqslant 1 \\
1-\eta^{2}, & |\eta| \geqslant 1
\end{array}\right\} .
$$

The computed normal tractions (35) cannot satisfy condition (16) unless

$$
\beta \geqslant-\{(1-A) /(2+A)\} \alpha,
$$

but conditions (34) and (37) can hold simultaneously only if

$$
\beta=-\{(1-A) /(2+A)\} \alpha, \quad \alpha \leqslant 0 .
$$

Then equation (32) becomes

$$
p^{\infty} / \mathscr{A}_{0}=1-\{(1-A) / 2(2+A)\} \alpha^{2},
$$

and the normal tractions reduce to

$$
\begin{gathered}
N(\eta)=-\mathscr{A}_{0} f(\eta)-\mathscr{A}_{0}\left|\frac{\eta-\beta}{\eta-\alpha}\right|^{A}\left\{\eta^{2}-\frac{3 A}{2+A} \alpha \eta-\frac{(1-A)(1+2 A)}{(2+A)^{2}} \alpha^{2}\right\}, \\
-\infty<\eta<\alpha \text { and } \beta<\eta<\infty
\end{gathered}
$$

With $|(\eta-\beta) /(\eta-\alpha)|^{A}$ expanded into a series of negative powers of $\eta$ it can be shown that $N( \pm \infty)=-p^{\infty}$. 
The case discussed is valid for $(\alpha, \beta)$ in the interval $(-1,1)$. The restriction that must be imposed on $p^{\infty} / \mathscr{A}_{0}$ is obtained from condition (29) by setting $-1<\alpha<0$ :

$$
3(1+A) / 2(2+A)<p^{x}: c_{0}<1 .
$$

\subsection{CASE II}

For pressures in the range

$$
0<p^{x_{0}} / \mathscr{A}_{0}<3(1+A) / 2(2+A)
$$

the separation zone extends heyond the trailing edge $\eta=-1$ of the incident pulse. In this case we could not obtain the integrals involved in a closed form and had to resort to a numerical computation.

It is convenient to use the dimensionless variables

$$
\xi=\delta u+\sigma, \quad \eta=\delta s+\sigma,
$$

where

$$
\delta=\frac{1}{2}(\beta-\alpha), \quad \sigma=\frac{1}{2}(\beta+\alpha),
$$

and retain the same symbols for the functions in the new variables. Thus equation (15) becomes

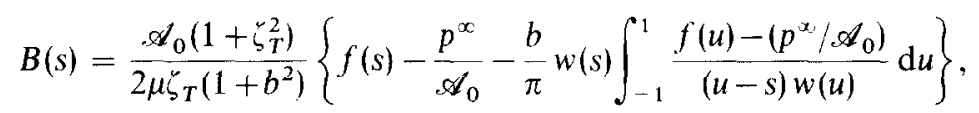

where

$$
w(s)=(1-s)^{A}(1+s)^{-A}
$$

From equation (26),

$$
\frac{p^{\infty}}{\mathscr{A}_{0}}=\frac{\sin \pi A}{2 \pi A} \int_{-1}^{1} \frac{f(u) \mathrm{d} u}{w(u)} .
$$

The integrals in equations (45) and (47) can be evaluated by means of the Gauss-Jacobi quadrature as extended for Cauchy singular integrals by Krenk [8]. The discretized forms of these equations are

$$
B\left(s_{i}\right)=-\frac{\mathscr{L}_{0}\left(1+\zeta_{T}^{2}\right)}{2 \mu \zeta_{T}\left(1+b^{2}\right)} \frac{b}{\pi} w\left(s_{i}\right) \sum_{k=1}^{n} A_{k}^{(n)} \frac{f\left(u_{k}\right)-\left(p^{\infty} / \mathscr{A}_{0}\right)}{u_{k}-s_{i}}: \quad(i=1,2, \ldots, n)
$$

and

$$
\frac{p^{\infty}}{\mathscr{A}_{n}}=\frac{\sin \pi A}{2 \pi A} \sum_{k=1}^{n} A_{k}^{(n)} f\left(u_{k}\right)
$$

where $A_{k}^{(n)}$ are the coefficients of the Gauss-Jacobi quadrature associated with the characteristic function $1 / w(s)$. Furthermore, $u_{k}$ and $s_{i}$ are the roots of the Jacobi polynomials

$$
\begin{aligned}
P_{n}^{(-A, A)}\left(u_{k}\right)=0 ; & (k=1,2, \ldots, n), \\
P_{n}^{(A,-A)}\left(s_{i}\right)=0 ; & (i=1,2, \ldots, n) .
\end{aligned}
$$


The discretized form of the normal tractions is

$$
N(s)=\mathscr{A}_{0}\left\{f(s)-\frac{p^{\infty}}{\mathscr{A}_{0}}-\frac{2 \zeta_{T} \mu}{\mathscr{A}_{0}\left(1+\zeta_{T}^{2}\right)} \frac{b}{\pi} \sum_{i=1}^{n} \frac{C_{i}^{(n)} B\left(s_{i}\right)}{\left(s_{i}-s\right) w\left(s_{i}\right)}\right\} ; \quad(|s|>1)
$$

where $C_{i}^{(n)}$ are the coefficients of the Gauss-Jacobi quadrature associated with the weight function $w(s)$.

The solution was obtained by iteration as follows. $\delta$ was chosen as the free parameter. With $\delta$ specified, a guess was made for $\sigma$, and $B\left(s_{i}\right)$ and $p^{\infty} / \varepsilon_{0}$ were obtained from expressions (48) and (49). In general, of course, the assumed $\sigma$ was different from its true valuc, say $\sigma_{0}$, and the inequalities (17) and (18) were not satisfied. Interpenetration appeared for $|\sigma|<\left|\sigma_{0}\right|$ and tensile tractions were encountered ahead of the leading edge $\eta=\beta$ of the separation zone for $|\sigma|>\left|\sigma_{0}\right|$. It was easy, however, to obtain lower and upper bounds for $\sigma_{0}$ and to make these bounds coincide for finding $\sigma_{0}$. Figure 1 shows the dependence of $\alpha$ and $\beta$ on $p^{\infty} / \mathscr{A}_{0}$ for $\theta_{0}=80^{\circ}$ and a material with $\lambda=\mu(\kappa=\sqrt{3})$. The results for Case $I$ were also recovered numerically and are included in the figure. $A$ representative distribution of the normal tractions is given in Figure 2 for $p^{\infty} \mathscr{A}_{0}=0.8$.

For values of the ratio $p^{\infty} / \mathscr{A}_{0}$ less than about 0.6 it was not possible to eliminate entirely the region of tensile tractions ahead of the leading edge under the present assumption of a single gap. This suggests that multiple gaps begin to appear as the ratio

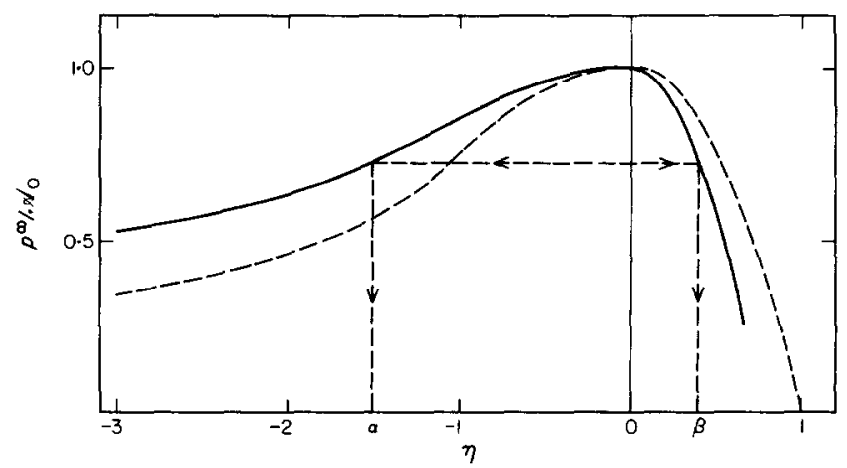

Figure 1. Dependence of $\alpha$ and $\beta$ on $p^{\infty} / \mathscr{A}_{0}$ for $\theta_{0}=80^{\circ}, \lambda=\mu$ (solid curve), and for the supersonic case [1] (dotted curve).

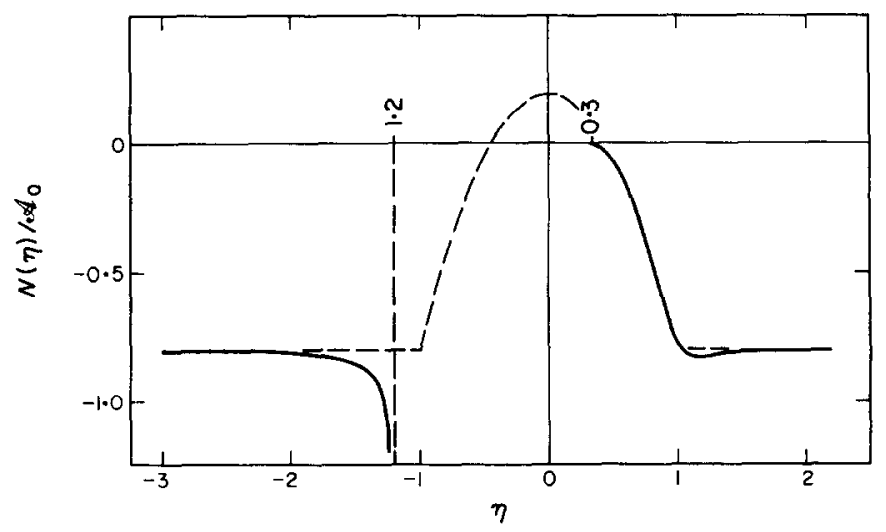

Figure 2. Normal tractions for $p^{\infty} / \mathscr{A}_{0}=0 \cdot 8, \theta_{0}=80^{\circ}$, and $\lambda=\mu$. The tractions in the bilateral problem are indicated by the dotted lines. 
$p^{*} / \mathscr{x}_{0}$ decreases. The normal tractions for $p^{\alpha} / \mathscr{A}_{0}=0.55$ are shown in Figure 3. The largest tension was encountered at $\eta=0.61$ for which $N / \mathscr{L}_{0}=0.007$.

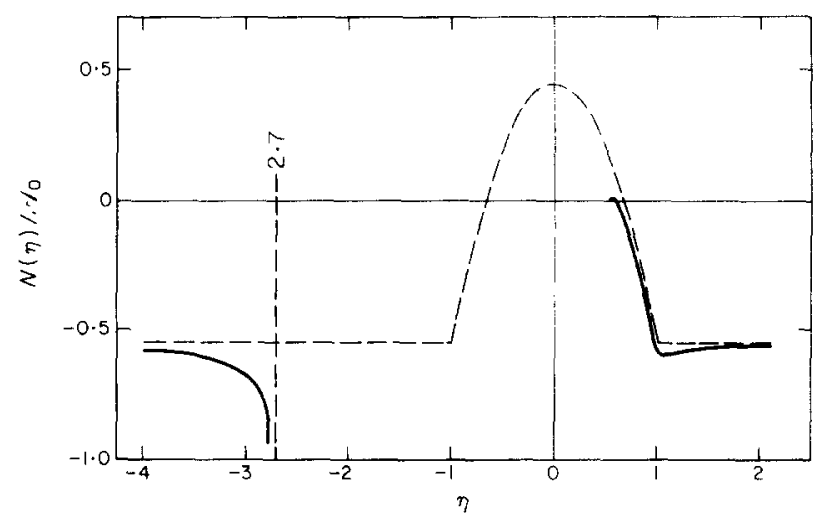

Figure 3. Normal tractions for $p^{\infty} / \mathscr{A}_{0}=0.55, \theta_{0}=80^{\circ}$ and $\lambda=\mu$. The tractions in the bilateral problem are indicated by the dotted lines.

\section{CONCLUSION}

An intriguing feature of the interaction between an incident elastic wave and a unilateral interface is the possible appearance of singularities at the trailing edges of the separation zones. This aspect of the interaction was first observed for incident harmonic waves or for a perfectly smooth input forcing the motion [3]. In the study of incident harmonic waves, we had to reason on the basis of a numerical solution that singularities appear under the conditions of total reflection. However, the present solution (Case I of the parabolic pulse) shows this explicitly. The jump discontinuities [2] and singularities [3] at the trailing edges of the gaps are obviously caused by the impact which takes place as the solids regain contact after separation, and the process resembles focusing.

The parabolic pulse is representative of a continuous wave that for relatively high levels of the applied pressure involves a single tension zone. One must also allow for the possibility of multiple, disconnected tension zones or for discontinuous pulses.

For $n$ multiple tension zones the integral equation (13) is modified to

$$
\begin{aligned}
B_{k}(\eta)+\frac{b}{\pi} \sum_{i=1}^{n} & {\left[\int_{\alpha_{i}}^{\beta_{i}} \frac{B_{i}(\xi)}{\xi-\eta} \mathrm{d} \xi\right]=\frac{\zeta_{T}^{2}+1}{2 \mu_{\zeta T}^{\zeta}}\left[\mathscr{A}_{0} f(\eta)-p^{\alpha}\right], } \\
\alpha_{k} & <\eta<\beta_{k}, \quad k=1,2, \ldots, n .
\end{aligned}
$$

Since in each separation interval only the $k$ th integral is singular, expression (53) actually represents a system of singular integral equations with generalized kernels. This system can be solved numerically by the methods described in reference [9] in conjunction with the side conditions

$$
\int_{\alpha_{k}}^{\beta_{k}} B_{k}(\xi) \mathrm{d} \xi=0, \quad k=1, \ldots, n .
$$

The formulation above can also be used for multiple separation zones that may appear even for a single tension pulse at relatively low applied pressures. In all cases. some 
judgment must be exercised, however, in the selection of the proper number of separation zones, since they do not necessarily equal the number of tensile humps present in the incident pulse.

The simplest case of a discontinuous pulse is the square pulse considered in reference [1]. For the square pulse, no matter what the level of $p^{\infty} / \mathscr{A}_{0}$, Case I will not occur, because $\alpha$ is always less than -1 . The interesting feature for this pulse is that logarithmic singularities may appear in $B(\eta)$ at the ends of the pulse -1 and 1 . According to Gakhov [10], expression (45) still remains valid, although numerical modifications will be required.

It is obvious from this discussion that the problem of the incident pulse is by no means exhausted. Any further investigation falls, however, outside the scope of the present paper.

\section{ACKNOWLEDGMENT}

One of the authors (M. C.) is pleased to acknowledge the support through the National Science Foundation grant ENG-7725032 during the course of this research.

\section{REFERENCES}

1. J. Dundurs and M. Comninou (1979) Wave Motion 1, 17-23. Interface separation caused by a plane elastic wave of arbitrary form.

2. M. Comninou and J. Dundurs 1977 Proceedings of the Royal Society, London A356, 509-528. Reflection and refraction of elastic waves in presence of separation.

3. M. Comninou and J. Dundurs (1978) Journal of Applied Mechanics 45, 548-552. Singular reflection and refraction of elastic waves due to separation.

4. F. G. FrIENDLANDER 1948 The Quarterly Journal of Mechanics and Applied Mathematics 1, 376-384. On the total reflection of plane waves.

5. L. BREKHOVSKIKH 1960 Waves in Layered Media. New York: Academic Press.

6. H. M. Berg, J. E. Bloom, H. Ishit, R. H. Marion, D. E. Pease, D. T. Spreng, J. B. VanderSande and J. WeERTMan 1970 in Fundamental Aspects of Dislocation Theory (edited by J. A. Simmons, R. deWit and R. Bullough), National Bureau of Standards Special Publication 317, Volume 1, 71-82. Subsonic, supersonic, and transonic dislocations moving on an interface separating two media of differing elastic properties.

7. N. I. MUSKHELISHVILI 1953 Singular Integral Equations. Groningen: Noordhoff.

8. S. KRENK 1975 Quarterly of Applied Mathematics 33, 225-232. On quadrature formulas for singular integral equations of the first and second kind.

9. F. ERdogan 1978 in Mechanics Today (editor S. Nemat-Nasser), Volume 4. New York: Pergamon Press. Mixed boundary value problems in mechanics.

10. F. D. Gakhov 1966 Boundary Value Problems. Oxford: Pergamon Press. 